\title{
Avaliação da Analgesia Pós-Operatória em Pacientes Submetidos à Cirurgia Orificial com Anestesia Local Associada ou Não à Morfina
}

\author{
Evaluation of the Postoperative Analgesia in Patients Submitted to Anorectal \\ Surgery with Local Anesthesia Associated or not the Morphine
}

\author{
JUVENALDAROCHATORRES NETO ${ }^{1}$, DANIELCARVALHO DE MENEZES ${ }^{2}$, ANACAROLINALISBOAPRUDENTE ${ }^{2}$, \\ JOARACOSTAALMEIDA² ${ }^{2}$ JORGE GONTRANTORRES DE MENEZES ${ }^{3}$ \\ ${ }^{1}$ Chefe do Serviço de Coloproctologia da Universidade Federal de Sergipe, ${ }^{2}$ Professor Doutor e coordenador do \\ internato de clínica cirúrgica do Serviço de Coloproctologia da Universidade Federal de Sergipe; ${ }^{3}$ Anestesiologista da \\ Universidade Federal de Sergipe - Sergipe - Brasil.
}

NETO JRT, MENEZES DC, PRUDENTE ACL, ALMEIDA JC, MENEZES JGT. Avaliação da analgesia pós-operatória em pacientes submetidos à cirurgia orificial com anestesia local associada ou não à morfina. Rev bras Coloproct, 2007;27(1): $037-041$.

RESUMO: Ainda não esta comprovada a eficácia dos derivados morfínicos ao nível de receptores opióides periféricos. Estudos procuram demonstrar o poder da droga em interferir na intensidade da dor quando infiltrada em nervos periféricos. Avaliamos, então, a infiltração local de morfina associada à anestesia local em cirurgias orificiais proctológicas. Nesse estudo foram analisados 61 pacientes, independentemente do gênero, sendo divididos aleatoriamente em dois grupos: a um grupo foi associada morfina ao anestésico local enquanto ao outro houve a administração do anestésico local sem a droga morfínica. Os pacientes de ambos os grupos foram submetidos à sedação e analgesia pós-operatória padronizadas. Foram avaliados: a intensidade da dor, a analgesia pós-operatória e a morbidade. A intensidade da dor, no momento de seu surgimento, foi semelhante nos dois grupos; o tempo de analgesia pós-operatória foi maior no grupo em que a morfina foi administrada, entretanto, não se mostrou estatisticamente significativo; as complicações pós-operatórias foram irrelevantes nos dois grupos. Dessa forma, a infiltração local de morfina na região anorretal tem benefícios em relação à analgesia pós-operatória que não mostraram significância estatística e não aumenta a incidência dos efeitos colaterais tão temidos relacionados às drogas morfínicas como retenção urinária e prurido.

Descritores: Morfina; analgesia; receptores opióides; nervos periféricos; anestesia local.

\section{INTRODUÇÃO}

A anestesia local em cirurgias proctológicas é exequível em aproximadamente $90 \%$ dos procedimentos, pode ser realizada em regime ambulatorial e reduz o custo em aproximadamente $50 \%{ }^{(3,4,5,6)}$. Essa técnica possibilita conforto ao paciente no pós-operatório $^{7}$, entretanto, busca-se uma analgesia mais eficaz.

Tradicionalmente é comprovada a eficácia dos derivados morfínicos associados aos anestésicos locais na obtenção de uma boa analgesia pós-operatória quan- do da realização de raquianestesia ou anestesia peridural ${ }^{(3)}$.

Trabalhos atuais procuram demonstrar a eficácia das drogas semelhantes à morfina ao nível de receptores opióides periféricos ${ }^{(8)}$.

O objetivo desse trabalho é avaliar, de forma prospectiva e comparativa, a infiltração local de morfina associada à anestesia local, em cirurgias orificiais proctológicas, relacionando o tempo de analgesia pósoperatória, a intensidade da dor e morbidade da técnica.

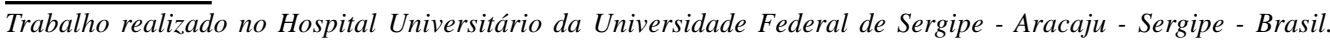

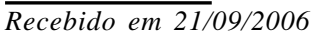

Aceito para publicação em 01/02/2007 


\section{PACIENTES E MÉTODOS}

Foram estudados 61 pacientes, adultos independentemente do gênero. Ao grupo A, de trinta pacientes, a anestesia local foi realizada com a administração de um volume total de trinta mililitros de marcaína a 0,33 por cento com adrenalina na diluição de 1/200.000. Ao grupo B, de trinta e um pacientes, a anestesia local foi realizada com a administração de um volume total de trinta mililitros de marcaína a 0,33 por cento com adrenalina na diluição de 1/200.000, acrescidos de 2 miligramas ( $\mathrm{mg}$ ) de morfina.

Todos os pacientes foram pré-medicados com midazolan na dose de $15 \mathrm{mg}$, por via oral, aos trinta minutos que precediam à cirurgia. Na sala de operação, anteriormente à realização da anestesia local de acordo com técnica padronizada, foram administrados, por via intravenosa, midazolan na dose de $0,03 \mathrm{mg} / \mathrm{kg}$ peso do paciente, alfentanil na dose de $15 \mathrm{ug} / \mathrm{kg}$ peso do paciente e propofol na dose de $1,5 \mathrm{mg} / \mathrm{kg}$ peso do paciente.

Aos pacientes foram disponibilizados todos os recursos mínimos aceitáveis de monitorização, pressão arterial média, oximetria de pulso, eletrocardiografia, capinografia, acrescidos de máscara venture com oxigênio a 100 por cento.

A intensidade da dor pós-operatória foi avaliada através de uma escala de 0 a 10 , onde 0 (zero) indica a ausência de dor e 10 caracteriza o extremo máximo de dor. Avaliamos também o tempo transcorrido entre o término da cirurgia e o início da dor, assim como o padrão da anestesia e a morbidade, referindose aos casos de retenção urinária, prurido e sangramento.

\section{RESULTADO}

No grupo sem morfina a idade variou entre 23 e 79 anos com média de 37,60. Foram realizados 79 procedimentos com média de 2,63 procedimentos por paciente. $\mathrm{O}$ padrão da anestesia foi ótimo em 27 pacientes $(90 \%)$ e bom em 3 pacientes $(10 \%)$. Dois pacientes $(6,67 \%)$ não apresentaram dor no pós-operatório imediato ( 24 horas). Nesse grupo a intensidade mínima da dor foi 2 e a máxima foi 10 , obtendo-se uma média de 5,57. Considerando-se os valores 5, 6 e 7 como de moderada intensidade na escala de dor, 10 pacientes $(33,33 \%)$ apresentaram dor leve, 12 pacientes (40\%) apresentaram dor moderada e 6 pacientes $(20 \%)$ apresentaram dor elevada.
No grupo sem morfina, dentre os pacientes que apresentaram dor no pós-operatório, o menor tempo de analgesia foi de 2 horas e o máximo de 24 horas obtendo-se uma média de 8 horas 49 minutos e 12 segundos. Nesse grupo 11 pacientes $(36,67 \%)$ apresentaram dor em um período inferior a 6 horas, 10 pacientes $(33,33 \%)$ apresentaram dor entre 6 e 10 horas de pós-operatório, 4 pacientes $(13,33 \%)$ apresentaram dor entre 10 e 15 horas do término da cirurgia e 5 pacientes $(16,67 \%)$ apresentaram dor depois de transcorridas 15 horas do ato cirúrgico.

No grupo sem morfina houve um caso $(3,33 \%)$ de retenção urinária.

No grupo com morfina a idade variou entre 17 e 56 anos com média de 35,23 . Foram realizados 86 procedimentos com média de 2,77 procedimentos por paciente. $O$ padrão da anestesia foi ótimo em 28 pacientes $(90,32 \%)$ e bom em 3 pacientes $(9,68 \%)$. Um paciente $(3,33 \%)$ não relatou dor no pós-operatório imediato ( 24 horas) e um paciente do grupo não pode ser avaliado, pois foi operado novamente. Nesse grupo, a intensidade mínima da dor foi 2 e a máxima foi 10, obtendo-se uma média de 5,34. Considerando-se os mesmos limites na escala de dor, 11 pacientes $(36,67 \%)$ apresentaram dor leve, 12 pacientes $(40 \%)$ apresentaram dor moderada e 6 pacientes $(20 \%)$ apresentaram dor elevada.

No grupo com morfina, dentre os pacientes que apresentaram dor, o menor tempo de analgesia foi de 3 horas e o máximo de 24 horas, obtendo-se uma média de 10 horas 38 minutos e 24 segundos. Nesse grupo 7 pacientes $(23,33 \%)$ apresentaram dor em um período inferior a 6 horas, 8 pacientes $(26,67 \%)$ apresentaram dor entre 6 e 10 horas de pós-operatório, 6 pacientes (20\%) apresentaram dor em um período entre 10 e 15 horas do término da cirurgia e 9 pacientes (30\%) apresentaram dor depois de transcorridas 15 horas do ato cirúrgico. de sangramento.

No grupo com morfina houve um caso $(3,23 \%)$

\section{DISCUSSÃO}

A técnica de anestesia local em cirurgias orificiais proctológicas demonstrou ser plenamente satisfatória, assim como no trabalho de CAPELHUCHINK et al., 2002, onde foi realizado hemorroidectomia com bloqueio da fossa ísquio retal com lidocaína associada ou não à morfina na dose de 1 $\mathrm{mg}{ }^{(9)}$. Esse fato pode ser evidenciado pelo padrão da 
anestesia obtido durante os procedimentos: o padrão foi ótimo em 55 pacientes $(90,16 \%)$ e bom em 06 pacientes $(9,84 \%)$, visto que houve a necessidade de administração de anestésico adicional para bloqueio locorregional (Figura 1), não havendo diferença significante entre os dois grupos. Não houve nenhum padrão de anestesia regular o que significa que a técnica de anestesia local não precisou ser substituída em nenhum momento por outra técnica anestésica como raquianestesia ou anestesia peridural, tanto no grupo em que foi utilizada a morfina como naquele onde a droga não foi administrada, fato relevante devido à maior possibilidade de complicações da anestesia ao nível da medula espinhal ${ }^{(10,11,12)}$. Os procedimentos em pacientes com afecções proctológicas realizados com anestesia local são, entretanto, mais delicados devido à complexa inervação da região que apresenta uma alta sensibilidade ${ }^{(13,14,15)}$, por isso busca-se uma analgesia mais eficaz. Com este objetivo busca-se demonstrar uma ação periférica dos opióides a qual esta sendo percebida na presença de inflamação ${ }^{(16)}$.

Houve baixa morbidade pós-operatória tanto no grupo em que houve a infiltração local de morfina como no grupo em que tal procedimento não foi realizado. Este fato pode ser demonstrado pelo baixo índice geral de complicações pós-operatórias, visto que foram registrados apenas um caso $(1,64 \%)$ de retenção urinária e um caso $(1,64 \%)$ de sangramento, um em cada grupo. A morfina foi o primeiro opióide natural a ser isolado em $1803^{(17)}$.

A analgesia pós-operatória também demonstrou ser satisfatória, tanto no grupo relacionado como no grupo não relacionado à morfina, no que se refere ao tempo transcorrido desde o fim do procedimento até o aparecimento da dor e em relação à intensidade desta quando do seu surgimento. Obtivemos para o primeiro um tempo médio de 10 horas, 38 minutos e 24 segundos e uma intensidade média da dor de 5,34 enquanto para o segundo foram transcorridos, em média, 8 horas, 49 minutos e 12 segundos até o surgimento da dor que apresentou uma intensidade média de 5,57 nesse momento. Além disso, três $(4,92 \%)$ pacientes não relataram dor no pós-operatório imediato (24 horas).

Houve um aumento considerável no tempo de analgesia pós-operatória no grupo em que houve a infiltração local de morfina quando comparado ao grupo em que esse procedimento não foi realizado (Figura 2), entretanto, não foi estatisticamente significativo (Tabela 1). Essa constatação, apesar de não ter obtido significância estatística, associada a outros estudos, demonstra que a infiltração periférica de morfina, durante cirurgia, produz analgesia eficaz, abalando o antigo princípio de que o poder analgésico dos opióides estaria relacionado, exclusivamente, a fenômenos no sistema nervoso central $^{(18)}$. Foi constatado um aumento médio no tempo de analgesia de 1 hora 49 minutos e 12 segundos, o que esta de acordo com estudos recentes, os quais relatam ser o acesso de opióides aos recepto-

\section{Padrão de Anestesia}

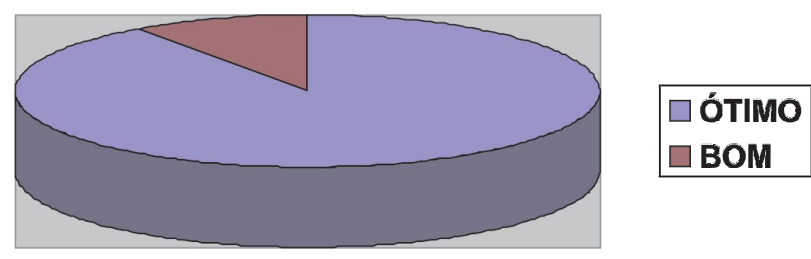

Figura 1 - Padrão da anestesia local em cirurgias orificiais proctológicas.

Azul - Padrão de anestesia ótimo - não houve necessidade de administração de uma quantidade extra de anestésico local.

Vermelho - Padrão de anestesia regular - houve necessidade de administração de uma quantidade extra de anestésico local.

\section{Tempo de Analgesia}

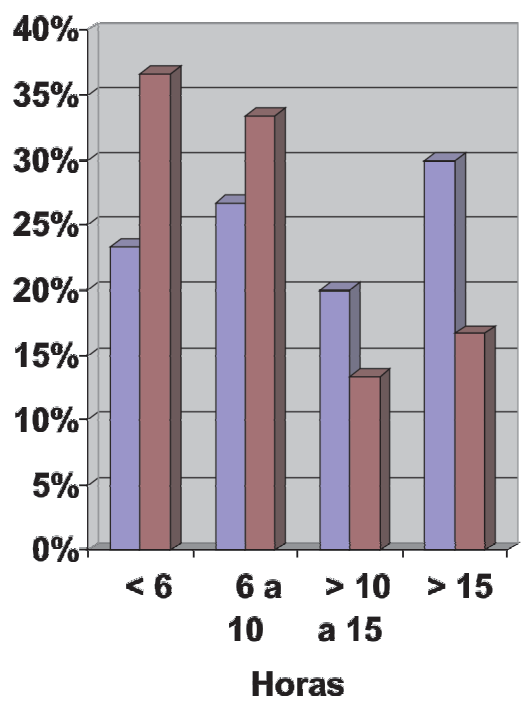

Com morfina Sem morfina

Figura 2 - Tempo de analgesia pós-operatória - intervalo de tempo, em horas, livre de dor nos diferentes grupos. O número, em cada intervalo, é expresso de acordo com a porcentagem de pacientes em relação ao seu grupo específico, seja aquele em que a morfina foi utilizada ou aquele em que somente o anestésico local foi administrado.

Azul-Pacientes que pertencem ao grupo em que houve a infiltração local de morfina associada ao anestésico local.

Vermelho - Pacientes que pertencem ao grupo em que somente o anestésico local foi utilizado. 
Tabela 1 - Analgesia pós-operatória.

\begin{tabular}{lcc}
\hline $\begin{array}{l}\text { Tempo de } \\
\text { analgesia }\end{array}$ & Com morfina & Sem morfina \\
\hline$<6 \mathrm{~h}$ & 7 & 11 \\
$6 \mathrm{~h}-10 \mathrm{~h}$ & 8 & 10 \\
$10-15 \mathrm{~h}$ & 6 & 4 \\
$>15 \mathrm{~h}$ & 9 & 5 \\
\hline
\end{tabular}

$P=0,26-$ Mann-Whitney $U$ Test.

res periféricos facilitado pela inflamação por ruptura do perineuro, uma cobertura normalmente quase impermeável que recobre as fibras nervosas periféricas ${ }^{(19)}$.

No que se refere à intensidade da dor no momento de seu surgimento (Figura 3 ) o benefício da morfina não foi expressivo (Tabela 2) havendo uma diminuição de apenas 0,23 na intensidade média, semelhante ao ocorrido no trabalho de CAPELHUCHINK et al., $2002^{(7)}$.

As complicações pós-operatórias não demonstraram estar relacionadas à infiltração local de morfina visto que nesse grupo não ocorreu nenhum caso de retenção urinária ou prurido e ocorreu apenas um caso $(3,23 \%)$ de sangramento, o qual não parece estar relacionado a drogas morfínicas.

\section{CONCLUSÕES}

A infiltração local de morfina na região anorretal não influenciou na intensidade da dor pós-operatória.

O tempo de analgesia pós-operatória foi ampliado com a infiltração de morfina, entretanto, não mostrou significância estatística.

A morbidade foi extremamente baixa e não esteve relacionada ao uso de morfina associada à anestesia local.

\section{Intensidade da Dor}

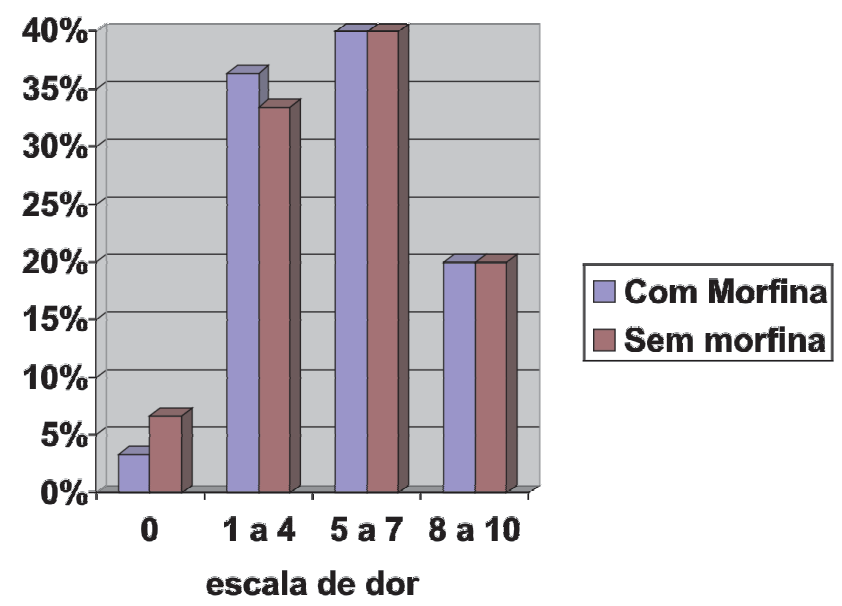

Figura 3 - Intensidade do primeiro episódio de dor - nível de sofrimento referido pelo paciente de acordo com uma escala padrão de dor. A intensidade foi assinalada referindo-se ao primeiro episódio de dor referido pelo paciente, sendo enquadrada, caso fosse relatada, em diferentes intervalos de acordo com os graus leve, moderado ou grave. O número, em cada intervalo, é expresso de acordo com a porcentagem de pacientes em relação ao seu grupo específico, seja aquele em que a morfina foi utilizada ou aquele em que somente o anestésico local foi administrado.

Azul-Pacientes que pertencem ao grupo em que houve a infiltração local de morfina associada ao anestésico local.

Vermelho - Pacientes que pertencem a grupo em que somente o anestésico local foi utilizado.

Tabela 2 - Intensidade da dor.

\begin{tabular}{lcc}
\hline $\begin{array}{l}\text { Intensidade } \\
\text { da dor }\end{array}$ & Com morfina & Sem morfina \\
\hline Ausente / leve & 12 & 12 \\
Moderada & 12 & 12 \\
Forte & 6 & 6 \\
\hline
\end{tabular}

$P=0,97-$ Mann-Whitney U Test.

ABSTRACT: It has not been proved the efficacy of morphine derived at periphery opium receivers. Studies are trying to demonstrate the power of the drug to interfere in the intensity of surgical pain while infiltrating in the periphery nerves. This study evaluated the infiltration of morphine associated with local anesthesia in anorectal surgery. Sixty one patients were analyzed, male and female, divided in two groups: in one group was associated morphine in the local anesthesia while in the other group only the local anesthetic was used. The patients of both groups were submitted to the same protocol standardized sedative during the surgery and postoperative analgesia. The intensity of pain was evaluated when it appeared, the time with analgesia was analyzed and other complications were registered. The intensity of pain was similar in both groups, the time with analgesia was longer in the group where morphine was used, although it was not significant in the statistics and the complications after the surgery were not significant in both groups. So the infiltration of morphine in the anorectal region has benefices in the postoperative analgesia but it was not significant in the statistics and it does not increase the complications related with morphine as urinary retention and itching.

Key words: Morphine; analgesia; opium receivers; nerves of periphery, local anesthesia. 


\section{REFERÊNCIAS}

1. Quilici FA, editor. Doenças Anorretais. $1^{a}$ Ed. São Paulo: Lemos Editorial; 2002

2. Sobrado CW, Nanhas SC, Marques CF, Habr-gama A - Cirurgia ambulatorial sob anestesia local em proctologia: experiência e análise do resultado de 503 operações. Rev bras Coloproct 2001; 21(4): p 228-233

3. Wetchler BV Anestesia Ambulatorial. In: Stoelting B C, editor. Tratado de Anestesiologia Clínica. 1a Ed. São Paulo: Manole; 1993. p 1619-1666

4. Adams AP, Cashman J, editors. Anestesia, Analgesia e Tratamento Intensivo. Rio de Janeiro: Revinter, 1994

5. Hanania M Tratamento da dor pós-operatória. In: Kanner R, editor. Segredos em clínica de dor. 1a Ed. Porto Alegre: Artes Médicas Sul; 1998. p 136-142

6. Lopes MB, Sousa LR, Porsani DF, Amaral AGV, Boas JrAV, Brishta SR - Associação de bupivacaína e morfina intra-articular para analgesia pós-operatória em cirurgia artroscópica de joelho. Revista brasileira de anestesiologia 1999; 49 (3): p 165-168

7. Capelhuchink P, Ju LY, Carvalho F, Ojeda CV, Bin FC, Klug WA - Hemorroidectomia ambulatorial com bloqueio da fossa isquiorretal com lidocaína e/ou morfina. Rev bras Coloproct 2002; 22(2): p 77-81

8. Murphy TM Anestesia Espinal, Epidural e Caudal. In: Miller RD, editor. Tratado de Anestesia. $2^{\mathrm{a}}$ Ed. São Paulo: Manole; 1989. p 1083-1134

9. Ganem EM Complicações Neurológicas da Anestesia Peridural e Subaracnóide. In: Cavalcanti IL, Cantinho FAF, Assad A, editors. Medicina Perioperatória. Rio de Janeiro: SAERJ; 2006. P 195-201
10. Hsieh JR, Hui YL, Yu CC, Lau WM, Ng YT, Wang YL - Local supplementation of ketoprofen reduces the incidence of low back pain after lumbar epidural anesthesia. Chaggeng Yi Xue Za Zhi, 1999; 22(3): p 439-444

11. Godoy JA; Godoy RS - Dor anal e retal. São Paulo: Arq. Gastroenterol, 1992; 29(4): p 153-160

12. Gabrielli F, Cioffi V, Chiarelli M, Guttadauro A, Simone MD - Hemorrhoidectomy with posterior perineal block. Dis Colon Rectum 200; 43(6): p 809-812

13. Sobrado CW, Habr-gama A - Hook needle puncture: a new technique of local anesthesia for anorectal surgery. Dis Colon Rectum 39, 1996; p 1330-1331

14. Hatheway JA Opióides. In: Duke J, Rosenberg SG, editors. Segredos em anestesiologia. Porto Alegre: Artes Médicas Sul; 1997. p 94-101

15. Felipe MAN, editor. Química de Los Morfinomiméticos. In De La Neuroleptoanalgesia a La Anestesia Analgésica, 2nd. México: Salvat; 1980. p 9-40

16. Rang HP, Dale MM, Ritter JM, editors. Fármacos analgésicos. In: Farmacologia. 4a ed. Rio de Janeiro: Guanabara Koogan; 2001. p 485-505

17. Gozzani JL Fisiopatologia da dor. In: Cavalcanti IL, Gozzani JL, editors. Dor pós-operatória. 1a ed. Rio de Janeiro: SBA; 2004. p 14-37

\section{Endereço para correspondência:}

DANIEL CARVALHO DE MENEZES

Endereço: Avenida Beira Mar, número 460 / 1201 - Farolândia

Aracaju-SE

49032-000

Tel.: (79) 9977-0558 / (79) 2107-5023

e-mail:menezesdcc@hotmail.com 